\title{
Role of FoxO1 in regulating autophagy in type 2 diabetes mellitus (Review)
}

\author{
XIUDAN LI, TINGTING WAN and YANBO LI \\ Department of Endocrinology, First Affiliated Hospital of Harbin Medical University, \\ Harbin, Heilongjiang 150001, P.R. China
}

Received September 14, 2020; Accepted February 9, 2021

DOI: $10.3892 /$ etm.2021.10139

\begin{abstract}
Type 2 diabetes mellitus (T2DM) is a major chronic disease that is characterized by pancreatic $\beta$-cell dysfunction and insulin resistance. Autophagy is a highly conserved intracellular recycling pathway and is involved in regulating intracellular homeostasis. Transcription factor Forkhead box $\mathrm{O} 1$ (FoxO1) also regulates fundamental cellular processes, including cell differentiation, metabolism and apoptosis, and proliferation to cellular stress. Increasing evidence suggest that autophagy and FoxO1 are involved in the pathogenesis of T2DM, including $\beta$-cell viability, apoptosis, insulin secretion and peripheral insulin resistance. Recent studies have demonstrated that FoxO1 improves insulin resistance by regulating target tissue autophagy. The present review summarizes current literature on the role of autophagy and FoxO1 in T2DM. The participation of FoxO1 in the development and occurrence of T2DM via autophagy is also discussed.
\end{abstract}

\section{Contents}

1. Introduction

2. Autophagy and T2DM

3. FoxO1 and T2DM

Correspondence to: Professor Yanbo Li, Department of Endocrinology, First Affiliated Hospital of Harbin Medical University, 23 You Zheng Street, Harbin, Heilongjiang 150001, P.R. China

E-mail: liyanbo34567@163.com

Abbreviations: AMPK, adenosine monophosphate-activated protein kinase; Atg, autophagy-related gene; ERS, endoplasmic reticulum stress; FoxO1, Forkhead box O1; GLP-1, glucagon-like peptide-1; G6Pase, glucose-6-phosphatase; miR, microRNA; mTOR, mammalian target of rapamycin; Pdx1, pancreatic and duodenal homeobox 1; PEPCK, phosphoenolpyruvate carboxykinase; ROS, reactive oxygen species; SIRT1, sirtuin 1; T2DM, type 2 diabetes mellitus; 3-MA, 3-methyladenine

Key words: T2DM, autophagy, FoxO1, pancreatic $\beta$-cell, insulin resistance
4. Autophagy, FoxO1 and T2DM

5. Conclusions and future direction

\section{Introduction}

Currently, diabetes is one of the most common chronic diseases. Lifestyle changes, such as overeating and increasingly sedentary lifestyles, continue to increase the number of diabetic cases, particularly type 2 diabetes mellitus (T2DM) (1). T2DM results from insulin resistance, and the gradual loss of mass and function of pancreatic $\beta$-cells (2). Inhibition of the excessive apoptosis of islet $\beta$-cells is essential for the treatment of diabetes (3). Increasing evidence suggest that islet $\beta$-cell apoptosis, cell viability and insulin secretion play essential roles in the pathogenesis of T2DM (2-6). In addition, insulin resistance plays a key role in T2DM and is associated with metabolic syndrome (7). Autophagy, oxidative stress, endoplasmic reticulum stress (ERS) and inflammation can moderate islet $\beta$-cell dysfunction and insulin resistance and contribute to the progression of T2DM $(2,7)$. Although multiple signaling pathways such as adenosine monophosphate-activated protein kinase (AMPK)/sirtuin 1 (SIRT1), AMPK-mammalian target of rapamycin (mTOR) are involved in the pathogenesis $(8,9)$ of T2DM, due to the complexity of T2DM, its overall pathogenesis remains unclear.

Autophagy has three major subtypes, macroautophagy, microautophagy and chaperone-mediated autophagy. The present review focuses on macroautophagy, which is commonly referred to as autophagy (10). Autophagy is a highly conserved intracellular recycling degradation pathway that targets cytosolic components for lysosomal degradation, and also acts as a cell survival mechanism that promotes cellular homeostasis $(2,10)$. The degradation of cytosolic contents via autophagy involves several intricate signaling pathways, including inositol requiring kinase 1 (IRE1)tumor necrosis factor receptor associated factor 2 (TRAF2) and the AKT-TSC-mTOR pathway $(7,10)$. Autophagy is a complex process with multiple steps, including initiation, nucleation, elongation/completion and fusion/degradation (10). Furthermore, autophagy is characterized by the lysosomal degradation of cellular material, which is a process of subcellular membrane rearrangement that sequesters the cytoplasm, proteins (or other cellular materials) and organelles, forming 
the autophagosome (10). Under physiological conditions, autophagy protects cellular homeostasis and prevent cells from undergoing oxidative stress and inflammation (10). Autophagy can also respond to a series of stresses, including the deprivation of nutrients or growth factors, hypoxia and reactive oxygen species (ROS) $(2,10)$. Furthermore, autophagy is extensively involved in several diseases, such as cancer, metabolic diseases and nervous system diseases $(2,4,11,12)$. Autophagy has also been implicated in the pathophysiology of T2DM (2-5,7), including the regulation of pancreatic $\beta$-cell mass and function (2-5) and peripheral insulin resistance (7). In addition, multiple transcription factors contribute to T2DM by regulating autophagy, such as transcription factor Forkhead box O1 (FoxO1), SIRT1 and pancreatic and duodenal homeobox 1 (Pdx1) $(8,9)$. However, the role of autophagy in the pathophysiology of T2DM remains controversial.

As a member of the forkhead box protein family, FoxO1 regulates fundamental cellular processes, including cell differentiation, metabolism and cell cycle arrest (13-15). FoxO1 activity is regulated by phosphorylation, acetylation and ubiquitination $(13,14,16)$. FoxO1 is extensively expressed in several tissues, including the pancreas (13), skeletal muscle (17), adipose tissue (18) and the liver (19). Furthermore, FoxO1 is highly expressed in pancreatic $\beta$-cells, and increasing evidence suggest that FoxO1 regulates $\beta$-cell replication and differentiation (13,20-23). Several studies have suggested that FoxO1 is closely associated with T2DM $(9,14,16,24,25)$. In addition, FoxO1 is a multifunctional protein that regulates the insulin sensitivity of target tissues $(19,26,27)$.

The present review summarizes the pathophysiological pathways associated with autophagy and FoxO1 in T2DM. Furthermore, the participation of FoxO1 in the development and occurrence of T2DM via autophagy is discussed.

\section{Autophagy and T2DM}

Autophagy and T2DM. The characteristics of T2DM are insulin resistance and the dysfunction of pancreatic $\beta$-cells $(2,7,10)$. Multiple mechanisms contribute to pancreatic $\beta$-cell dysfunction and mass loss, and insulin resistance, including oxidative stress, ERS, upregulation of apoptosis and the uncontrolled autophagy of $\beta$-cells $(2,4,13,28)$. Previous studies have reported that autophagy regulates $\beta$-cell viability and apoptosis, and participates in the development and occurrence of T2DM (2-4,7). Dysregulation of autophagy can lead to the dysfunction of $\beta$-cells or abnormal insulin secretion in pancreatic $\beta$-cells (2-4).

The present review summarizes current literature on the role of autophagy in the survival and insulin secretion of $\beta$-cells, and insulin resistance, focusing on autophagy during the progression of T2DM.

Autophagy regulates $\beta$-cell viability and apoptosis. The regulation of pancreatic $\beta$-cell mass and function is vital for the pathogeny of T2DM. Furthermore, the viability of $\beta$-cells is crucial in the occurrence and development of T2DM. The number of $\beta$-cells decreases with an increase in the apoptotic rate (2-5). In addition, the extracellular accumulation of islet amyloid, derived from human islet amyloid polypeptide (hIAPP), also contributes to T2DM (29).
Previous studies have demonstrated that the inhibitor, 3-methyladenine (3-MA), pretreated with autophagy decreases the viability of INS-1 cells and promotes cell apoptosis via ROS-mediated inflammation $(3,4)$. Furthermore, increasing evidence suggest that certain drugs protect pancreatic $\beta$-cells by enhancing autophagy $(3,5,8)$. For example, glucagon-like peptide-1 (GLP-1) analogs, liraglutide and metformin, can suppress apoptosis by inducing autophagy in islet $\beta$-cells in a high-glucose environment $(3,8)$. In an in vivo study, knockdown of autophagy-related gene (ATG)7 in ob/ob mice increased the rate of apoptotic $\beta$-cells and decreased the $\beta$-cell mass, which resulted in severe DM (30). In addition, the treatment of female diabetic Akita mice with rapamycin-induced autophagy improved ER-induced diabetes and inhibited $\beta$-cell apoptosis (31). The autophagy deficiency in mice caused the accumulation of hIAPP and exacerbated the hIAPP-induced $\beta$-cell toxicity and apoptosis (29). Fan et al (5) suggested that liraglutide can promote pancreatic $\beta$-cell proliferation in a high-fat fed and streptozotocin-induced mouse model of T2DM by enhancing autophagy. Taken together, these findings confirm that autophagy is indispensable for the survival of islet $\beta$-cells.

Autophagy and insulin secretion. Dysfunction of insulin secretion is due to the abnormalities in insulin biosynthesis or degradation (32). During the early stages of T2DM, islet $\beta$-cells exhibit a compensated higher insulin release. However, as T2DM progresses, islet $\beta$-cells gradually exhibit impaired glucose-stimulated insulin secretion $(32,33)$. The deletion of free fatty acid receptor 3 enhances insulin secretion in mice; however, this is not due to the increase in $\beta$-cell mass (34). Thus, the underlying molecular mechanisms of impaired insulin secretion remain unclear.

Previous studies have demonstrated the essential roles of autophagy in T2DM development (3-5). Furthermore, autophagy plays an essential role in insulin homeostasis (32). Riahi et al (32) revealed that the lysosomal degradation of proinsulin through autophagy. Autophagy deficiency does not affect basal insulin secretion but increases glucose or $\mathrm{KCl}$-stimulated insulin secretion (32). A recent study reported that pretreatment with rapamycin, an mTOR inhibitor, induces autophagy and significantly decreases the insulin content in NIT-1 cells (35). Another study reported similar results, indicating that the level of insulin significantly increases due to glibenclamide, and further increases with 3-MA (36). Collectively, these findings suggest that suppressing autophagy increases $\beta$-cell insulin secretion. In an in vivo study, autophagy hyperactive mice exhibited impaired insulin secretion compared with mice with normal autophagy, the effects of which were reversed following transient treatment with autophagy inhibitors (37). This may be due to the theory that overexpression of autophagy increases the degradation of insulin granules in insulin-secreting $\beta$-cells $(32,37)$.

Conversely, several studies have suggested that deficiency of autophagy decreases the insulin secretion in pancreatic $\beta$-cells $(30,38,39)$. Mice with the $\beta$-cell specific deletion of ATG7, which is a necessary for autophagy (10), developed glucose intolerance that resulted from decreased insulin secretion $(30,38,39)$. Due to the insufficient unfolded protein response machinery, autophagy-deficient $\beta$-cells fail to adapt 
to the lipotoxicity environment, which decreases insulin secretion and diabetes in ATG7 deficiency mice (30). Jung et al (39) revealed that fasting serum insulin concentration is notably lower in the $\beta$-cell specific deletion of ATG7 mice compared with the control mice; however, the levels of ubiquitinated proteins increase.

Taken together, these findings suggest that autophagy has a dual effect with the insulin secretion of $\beta$-cells, but the underlying mechanisms still need further research.

Autophagy and insulin resistance. Insulin resistance has an important effect on the occurrence of T2DM and is closely associated with metabolic syndrome (40). Insulin resistance refers to the effect of insulin, which decreases in insulinresponsive tissues, including adipocytes, the liver and skeletal muscles $(7,40)$. In the liver, failure of insulin action decreases glycogen synthesis and increases gluconeogenesis. For adipose tissues, insulin-mediated glucose utilization markedly decreases, which results in high levels of free fatty acids in insulin-resistant states. The skeletal muscle shares $80 \%$ of the total insulin-stimulated glucose uptake in humans (7). Thus, insulin resistance in the skeletal muscle plays a significant role in the development of T2DM $(7,41)$.

Multiple factors cause insulin resistance in target tissues, such as mitochondrial dysfunction, ERS and inflammation (2,7). However, the underlying molecular mechanism of insulin resistance remains unclear. Increasing evidence suggest an association between autophagy and insulin resistance (41-44). Progranulin may mediate adipose insulin resistance, at least in part, by inducing autophagy through activation of oxidative stress and ERS (43). Autophagy-hyperactive mice have improved insulin sensitivity in insulin target tissues by decreasing ERS (5). The suppression of autophagy results in insulin resistance in the liver tissues of mice. Furthermore, restoration of autophagy in obese mice induces insulin action in liver tissues (28). Insulin receptor substrate-1 (IRS1) is the direct substrate of the insulin-sensing insulin receptor, which is closely associated with insulin resistance (7). ATG16L1 deficiency in mouse embryonic fibroblasts results in insulin resistance, which is mediated by IRS1 degradation (41). Collectively, these studies indicate that autophagy suppresses insulin resistance in insulin target tissues. Furthermore, these studies demonstrate that autophagy has a beneficial effect in regulating insulin resistance. Thus, the level of autophagy is important for tissue insulin sensitivity.

\section{FoxO1 and T2DM}

Increasing evidence suggest that FoxO1 regulates cell proliferation, apoptosis, differentiation, antioxidant stress and insulin secretion in pancreatic $\beta$-cells, and is closely associated with T2DM (13,24,25,45-48). Furthermore, FoxO1 plays a crucial role in regulating the insulin sensitivity of insulin target tissues $(17,19,27,49)$.

FoxOl regulates the survival of $\beta$ cells. The regulation of pancreatic $\beta$-cell survival is vital for the $\beta$-cell mass in T2DM $(3,4)$. FoxO1 plays a crucial role in $\beta$-cell survival $(50,51)$. Nak et al (18) reported that protein kinase C delta induces the nuclear accumulation of FoxO1 but does not increase $\beta$-cell death. Furthermore, FoxO1 does not induce pro-apoptotic genes but may exert beneficial effects when phosphorylated under non-stress environments (22). Inhibition of FoxO1 dephosphorylation is necessary for the induction of pro-apoptotic gene Bcl-2, which may explain why nuclear FoxO1 is not pro-apoptotic on pancreatic $\beta$-cells (22). However, whether downregulation or upregulation of FoxO1 affect the glucocorticoid receptor-induced $\beta$-cell apoptosis remains to be investigated (23).

Chronic oxidative stress is associated with cellular apoptosis (47). Furthermore, it has been demonstrated that oxidative stress is one of the mechanisms for $\beta$-cell dysfunction and failure by chronic exposure to elevate glucose concentrations (50). Zhang et al (51) reported that FoxO1 protects $\beta$-cells from oxidative stress by inducing the expression of $\beta$-cell antioxidant enzymes, such as superoxide dismutase, catalase and glutathione peroxidase 1. Furthermore, FoxO1 protects pancreatic $\beta$-cells against oxidative stress by suppressing the expression of the thioredoxin-interacting protein, which is a cytosolic factor that regulates $\beta$-cell apoptosis (52). Taken together, these findings suggest that FoxO1 plays a protective role in $\beta$-cell survival.

FoxOl regulates $\beta$-cell insulin secretion. Previous studies have demonstrated that FoxO1 is an important player in $\beta$-cell insulin secretion $(8,9,13,53-55)$. $\mathrm{Pdx} 1$ is a key regulator of $\beta$-cell differentiation, proliferation and insulin synthesis $(56,57)$. A previous study reported that FoxO1 inhibits Pdx1 expression, thereby offering a mechanism in which FoxO1 may regulate $\beta$-cell insulin secretion (24). Chronic exposure to high glucose in $\beta$-cells activates FoxO1 and increases its nuclear location, which in turn decreases Pdx1 expression and reduces insulin secretion (55). FoxO1 knockdown efficiently decreases microRNA (miR)-802 expression in Min6 cells in mice (58), while overexpression of miR-802 in $\beta$-cells impairs insulin synthesis and secretion (58). FoxO1 knock-in mice in the pancreas develops glucose intolerance and impairs insulin secretion due decreased Pdx1 expression (53). Inhibition of FoxO1 reverses the dexamethasone-induced impairment on insulin secretion in rat islets by inhibiting Pdx-1 expression (54).

Neurogenic differentiation factor (NeuroD) and Mus musculus v-maf muscu-loaponeurotic fibrosarcoma oncogene family protein A (MafA) are insulin gene transcription factors of $\beta$-cells that promote insulin secretion $(20,56)$. In cultured $\beta \mathrm{TC}-3$ cells, FoxO1 stimulates NeuroD and MafA expression in response to oxidative damage induced by $\mathrm{H}_{2} \mathrm{O}_{2}$ treatment, and preserves islet function (20). This concept was confirmed by a study that revealed that FoxO1-transgenic mice exhibit significantly elevated insulin 1 gene expression levels, accompanied by upregulation of pancreatic Pdx1 and NeuroD (51). However, db/db mice lack FoxO1, particularly in pancreatic $\beta$-cells, which decreases insulin secretion and exhibits severe glucose intolerance (21). This suggests that FoxO1 is necessary for the maintenance of insulin secretion under metabolic stress (21). Collectively, these findings indicate that FoxO1 plays an essential role in insulin secretion.

FoxO1 regulates insulin resistance. FoxO1 is extensively expressed in insulin target tissues, such as hepatocytes, 


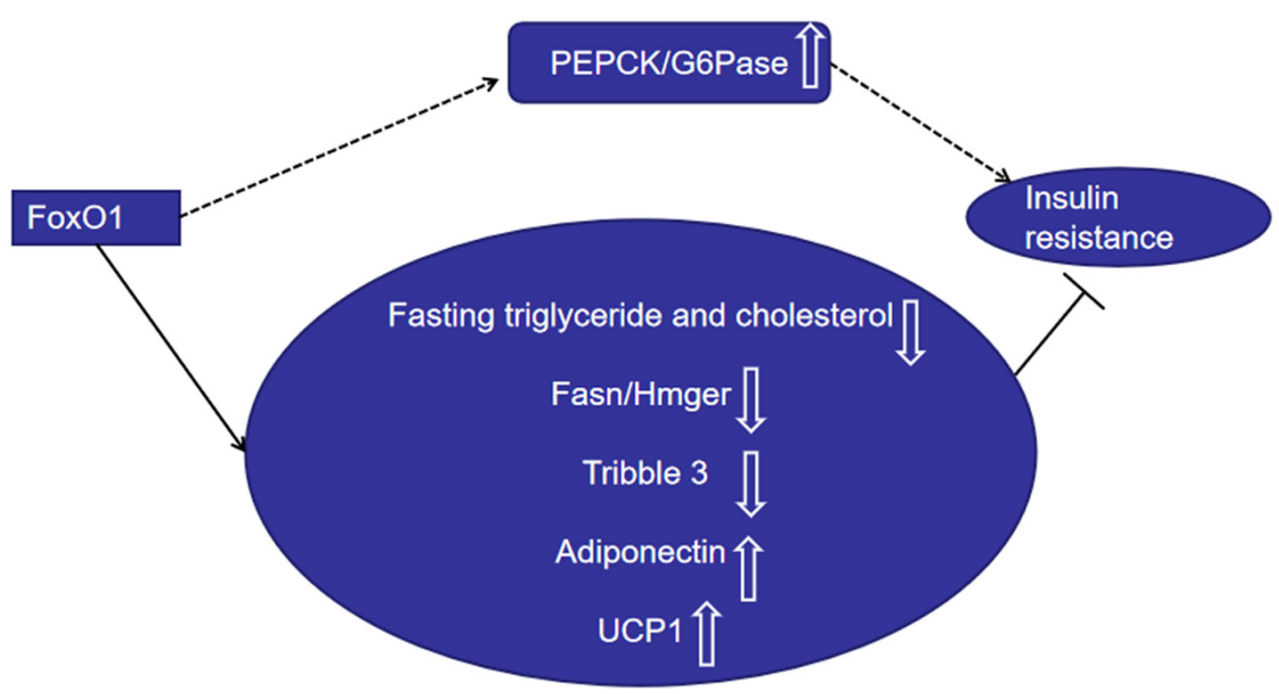

Figure 1. FoxO1 promotes insulin resistance by upregulating PEPCK and G6Pase expression. FoxO1 inhibits insulin resistance by downregulating fasting triglyceride and cholesterol levels, and suppressing Fasn/Hmger and tribble 3 expression, while upregulating adiponectin and UCP-1 expression. FoxO1, Forkhead box O1; PEPCK, phosphoenolpyruvate carboxykinase; G6Pase, glucose-6-phosphatase; UCP1, uncoupling protein 1.

adipocytes and skeletal muscles (17-19). Previous studies have proposed that FoxO1 plays a significant role in the occurrence and development of insulin resistance $(18,59,60)$. However, FoxO1 also promotes insulin resistance, which has been demonstrated in several studies $(17,19,27,49)$. FoxO1 knockdown in HepG2-insulin resistance cells decreases the glucose content and alleviates insulin resistance in primary hepatocytes (22). Phosphoenolpyruvate carboxykinase (PEPCK) and glucose-6-phosphatase (G6Pase) are the two key enzymes of gluconeogenesis $(19,49)$. A recent study revealed that inhibiting FoxO1 expression significantly decreases the blood glucose content and mRNA level of PEPCK/G6Pase in HepG2 cells (50). Gu et al (19) supported these results, suggesting that FoxO1 inhibits PEPCK and G6Pase expression, and glucose output in hepatocytes. In addition, it has been reported that FoxO1 expression decreases in the liver of T2DM insulin resistance mice induced by high-fat diet (19). Inhibiting FoxO1 mRNA expression with antisense oligonucleotides therapy in mice improves the clearance of glucose following intraperitoneal glucose load, and improves the hepatic insulin sensitivity and adipocyte insulin action (27). Mice specifically overexpressing FoxO1 in the skeletal muscle exhibit impaired glucose tolerance and insulin tolerance (17), suggesting that FoxO1 promotes insulin resistance and muscle atrophy (17).

Some studies have indicated that FoxO1 is a counterregulator of target tissue insulin resistance (18,26,59-63). Dysregulation of lipid metabolism in the liver contributes to insulin resistance $(63,64)$. Fasting triglyceride and cholesterol levels, and postprandial triglyceride levels are substantially suppressed in transgenic mice that express active FoxO1 (63). Zhang et al (64) reported similar results, indicating that hepatic deletion of FoxO1 increases cholesterol concentrations and serum triglyceride and elevates hepatic lipid secretion. Furthermore, overexpression of FoxO1 inhibits the expression levels of lipogenesis genes, including Fasn and Hmgcr (64). The expression of pseudokinase tribble 3 has been reported to negatively regulate insulin sensitivity $(60,65)$. FoxO1 suppresses tribble 3 expression in an insulin-dependent manner in hepatocytes and promotes insulin sensitivity during fasting (60). Phosphorylated mammalian FoxO1 suppresses hepatic glucose production (66). Adiponectin is a hormone that plays a key role in regulating energy homeostasis and metabolism $(67,68)$. Furthermore, adiponectin plays a crucial role in improving insulin sensitivity in adipose tissues, the liver and muscles, and exerts antidiabetic functions $(67,68)$. GLP-1 agonist exendin-4 induces adiponectin expression (69). However, suppression of Foxo-1 impairs the effect of exendin-4 on the adiponectin expression in 3T3-L1 adipocytes (69). Overexpression of mutant FoxO1 in brown adipose tissues increases the number of brown adipocytes and the expression of the uncoupling protein 1 (UCP1) gene in T37i cells (18). FoxO1 protects against insulin resistance during adipocyte differentiation (26). FoxO1 transgenic mice increased insulin sensitivity in adipose tissue (60). FoxO1 deletion decreases IRS2 expression, glucose uptake and insulin action in muscle tissues (62).

Collectively, these findings suggest that FoxO1 has a dual role in regulating insulin resistance (Fig. 1). In addition to regulating $\beta$-cell mass, FoxO1 also regulates $\beta$-cell function, and the level of FoxO1 is important for tissue insulin sensitivity.

\section{Autophagy, FoxO1 and T2DM}

FoxO1-autophagy axis. FoxO transcription factors regulate two main proteolytic systems, the ubiquitin-proteasome and autophagy-lysosome systems (70). Increasing evidence suggest that FoxO1 upregulates the expression of autophagy (71-73). FoxO1 elicits autophagy in a transcription-independent manner (72). Acetylated FoxO1 interacts with endogenous ATG7 and induces autophagy and suppresses cell viability in cancer cells (72). Liu et al (73) reported that FoxO1 antagonists suppress autophagy during adipocyte differentiation and decrease lipid droplet size in adipocytes. FoxO1 enhances autophagy, and the FoxO1-autophagy axis regulates lipid droplet growth in white adipose tissues (73). Notably, activation of FoxO1 increases Rab7 expression, while the 
accumulation of p62 decreases in vascular endothelial cells treated with ox-LDL (11). Treatment with FoxO1 small interfering RNA inhibits autophagic flux, resulting in oxidative stress and apoptosis in human cholangiocarcinoma QBC939 cells (12). A recent study reported that metformin alleviates renal injury in diabetic rats by regulating the adenosine monophosphate-activated protein kinase-SIRT1-FoxO1 pathway, thus promoting autophagy (74). The inhibitory effect of miR-21 on the expression of the autophagy-related protein, LC3II/I, and the facilitation of p62 is abrogated following upregulation of FoxO1 in high glucose-cultured podocytes (75). The inhibitor of FoxO1 transcriptional activity, AS, decreases autophagic activity and cell death of cardiomyocyte $\mathrm{H} 9 \mathrm{c} 2$ cells. Both in vivo and in vitro experiments have demonstrated that 1,25-dihydroxyvitamin $\mathrm{D}$ attenuates diabetic heart-related cardiac autophagy and damage by activating vitamin D to suppress the nuclear translocation of FoxO1 (76). Activation of FoxO1 in cardiomyocytes decreases the cell size and significantly increases the expression levels of LC3 and ATG12 (77). FoxO1 silencing markedly decreases autophagy induced by oxidative stress and increases apoptosis in cardiomyocytes (78). Resveratrol enhances the autophagic flux via the SIRT1/FoxO1/Rab7 axis, and ameliorates myocardial oxidative stress injury in diabetic mice (79). Taken together, these findings suggest that FoxO1 promotes autophagy in cardiomyocytes. Platelet-rich plasma decreases apoptosis in human osteoarthritic cartilage by increasing FoxO1 and autophagy (80). Autophagy inhibitor, 3-MA, significantly aggravates high-glucose-induced apoptosis and decreases cell viability in cardiac myocytes (81). In addition to decreasing the autophagy-related protein, LC3II/I and cell viability, FoxO1 silencing also enhances the high-glucose-mediated apoptosisrelated protein caspase-3 activation (81). Collectively, these results suggest that FoxO1 inhibits apoptosis and enhances cell survival by promoting autophagy.

FoxOl-autophagy axis and T2DM. Autophagy and FoxO1 are both involved in the development of T2DM $(2,10,13,14)$. Autophagy has a crucial effect on $\beta$-cell mass and function, and target tissue insulin resistance $(2,7,10,44)$. Previous studies have reported that moderate autophagy can promote $\beta$-cell survival, insulin secretion and target tissue insulin sensitivity $(2,3,7,10)$. Furthermore, it has been demonstrated that antidiabetic agents, liraglutide and metformin, promote islet $\beta$-cell proliferation and suppress $\beta$-cell apoptosis by enhancing autophagy $(5,8)$.

Increasing evidence suggest that the FoxO1-autophagy axis is involved in several diseases, including diabetes, cardiovascular disease and cancer $(11,12,15,74,77)$. FoxO1 has been demonstrated to upregulate cell autophagy in different types of cells, including cancer cells (72), adipocytes (73), podocytes (75) and cardiomyocytes (78). FoxO1 is essential for the progression of T2DM, although the role of FoxO1 in $\beta$-cell function and mass remains controversial. Increasing evidence suggest that FoxO1 improves insulin resistance via autophagy in T2DM $(82,83)$. Inflammatory factors, such as interleukin-6 (IL)-6, tumor necrosis factor- $\alpha$ (TNF- $\alpha$ ) and nod-like receptor 3 (NLRP3), contribute to the insulin resistance of pancreatic islets in T2DM (4). Upregulation of FoxO1 enhances autophagy activity and decreases the expres- sion levels of the inflammatory cytokines, IL-6, TNF- $\alpha$ and NLRP3, in the liver (82). The FoxO1-mediated transcription of key autophagy genes is an intricate part of insulin suppression of autophagy in cultured hepatocytes (82). FoxO1 deficiency impairs autophagy in the liver, and overexpression of FoxO1 stimulates ATG14 gene expression in mouse primary hepatocytes and lowers triglyceride concentrations in mice (83). miR-378 inhibits apoptosis by promoting autophagy in skeletal muscles and sustains autophagy through FoxO1-mediated transcriptional reinforcement by targeting phosphoinositidedependent protein kinase 1 (80). FoxO1 activation induces lipid droplet degradation via autophagy and improves metabolic stress in adipocytes (84).

Increasing evidence suggest that FoxO1-mediated autophagy enhances cell viability and inhibits cell apoptosis in different types of cells $(12,79,80,81)$. However, the effect of FoxO1 on autophagy in pancreatic $\beta$-cells requires further investigation.

Taken together, current literature suggest that FoxO1 silencing inhibits autophagy of INS-1 cells and decreases the survival of INS-1 cells (unpublished data). In conclusion, the FoxO1-autophagy axis is closely associated with T2DM.

\section{Conclusions and future direction}

T2DM poses a serious public-health threat to those affected. Autophagy and FoxO1 are involved in pancreatic $\beta$-cell dysfunction and insulin resistance. Thus, regulation of FoxO1 and autophagy activity in islet $\beta$-cells has the potential to serve as a novel therapeutic strategy against T2DM. In addition, some hypoglycemic drugs, such as metformin, GLP-1 receptor agonists and GLP-1 analogs, have been demonstrated to exert anti-T2DM effects via autophagy and FoxO1. However, further studies are required to determine whether these drugs can achieve the effect of improving $\beta$-cell function and mass, and insulin resistance via the FoxO1-autophagy axis.

\section{Acknowledgements}

Not applicable.

\section{Funding}

The present review was supported by the National Natural Science Foundation of China (grant no. 81770820).

\section{Availability of data and materials}

Not applicable.

\section{Authors' contributions}

All authors contributed equally to the conception and design of the present review, and drafted and revised the initial manuscript for important intellectual content. All authors have read and approved the final manuscript.

\section{Ethics approval and consent to participate}

Not applicable. 


\section{Patient consent for publication}

Not applicable.

\section{Competing interests}

The authors declare that they have no competing interests.

\section{References}

1. Harris SR, Carrillo M and Fujioka K: Binge-eating disorder and type 2 diabetes: A Review. Endocr Pract: December 20, 2020. doi: 10.1016/j.eprac.2020.10.005.

2. Wang Y, Li YB, Yin JJ, Wang Y, Zhu LB, Xie GY and Pan SH: Autophagy regulates inflammation following oxidative injury in diabetes. Autophagy 9: 272-277, 2013.

3. Chen ZF, Li YB, Han JY, Yin JJ, Wang Y, Zhu LB and Xie GY: Liraglutide prevents high glucose level induced insulinoma cells apoptosis by targeting autophagy. Chin Med J (Engl) 126: 937-941, 2013.

4. Zhu LB, Cao MM, Wang J, Su Y, Jiang W, Liu GD and Li YB: Role of autophagy in LPS-induced inflammation in INS-1 cells. Mol Med Rep 19: 5211-5218, 2019.

5. Fan M, Jiang H, Zhang Y, Ma Y, Li L and Wu J: Liraglutide enhances autophagy and promotes pancreatic $\beta$ cell proliferation to ameliorate type 2 diabetes in high-fat-fed and streptozotocintreated mice. Med Sci Monit 24: 2310-2316, 2018.

6. Melmed L and Polonsky KS, Reed Larsen P and Kronenberg H: Chapter on pathogenesis of type 2 diabetes-Williams Textbook of Endocrinology. 13th edition. Elsevier, 2016.

7. Zhang N, Cao MM, Liu H, Xie GY and Li YB: Autophagy regulates insulin resistance following endoplasmic reticulum stress in diabetes. J Physiol Biochem 71: 319-327, 2015.

8. Li Q, Jia S, Xu L, Li B and Chen N: Metformin-induced autophagy and irisin improves INS-1 cell function and survival in high-glucose environment via AMPK/SIRT1/PGC-1 $\alpha$ signal pathway. Food Sci Nutr 7: 1695-1703, 2019.

9. Cheng STW, Li SYT and Leung PS: Fibroblast growth factor 21 stimulates pancreatic islet autophagy via inhibition of AMPK-mTOR signaling. Int J Mol Sci 20: 2517. doi: 10.3390/ ijms20102517.

10. Lee YH, Kim J, Park K and Lee MS: $\beta$-cell autophagy: Mechanism and role in $\beta$-cell dysfunction. Mol Metab 27S: S92-S103, 2019.

11. Wu Q, Hu Y, Jiang M, Wang F and Gong G: Effect of autophagy regulated by Sirt1/FoxO1 pathway on the release of factors promoting thrombosis from vascular endothelial cells. Int J Mol Sci 20: 4132, 2019. doi: 10.3390/ijms20174132.

12. He W, Zhang A, Qi L, Na C, Jiang R, Fan Z and Chen J: FOXO1, a potential therapeutic target, regulates autophagic Flux, oxidative stress, mitochondrial dysfunction, and apoptosis in human cholangiocarcinoma QBC939 cells. Cell Physiol Biochem 45: $1506-1514,2018$

13. Kitamura T: The role of FOXO1 in $\beta$-cell failure and type 2 diabetes mellitus. Nat Rev Endocrinol 9: 615-623, 2013.

14. Xing YQ, Li A, Yang Y, Li XX, Zhang LN and Guo HC: The regulation of FOXO1 and its role in disease progression. Life Sci 193: 124-131, 2018.

15. Cheng Z: The FoxO-autophagy axis in health and disease. Trends Endocrinol Metab 30: 658-671, 2019.

16. Chen B, Zhou W, Zhao W, Yuan P, Tang C, Wang G, Leng J, Ma J, Wang X, Hui Y, et al: Oxaliplatin reverses the GLP-1R-mediated promotion of intrahepatic cholangiocarcinoma by altering FoxO1 signaling. Oncol Lett 18: 1989-1998, 2019.

17. Kamei Y, Miura S, Suzuki M, et al. Skeletal muscle FOXO1 (FKHR) transgenic mice have less skeletal muscle mass, downregulated Type I(slow twitch/red muscle) fiber genes, and impaired glycemic control. J Biol Chem. 2004;279(39):41114ロ41123. doi:10.1074/jbc.M400674200

18. Nakae J, Cao Y, Oki M, et al. Forkhead transcription factor FoxO1 in adipose tissue regulates energy storage and expenditure. Diabetes. 2008;57(3):563ロ576. doi:10.2337/db07-0698
19. Gu L, Ding X, Wang Y, et al. Spexin alleviates insulin resistance and inhibits hepatic gluconeogenesis via the FoxO1/PGC-1a pathway in high-fat-diet-induced rats and insulin resistant cells. Int J Biol Sci. 2019;15(13):2815口2829. Published 2019 Nov 1. doi:10.7150/ijbs.31781

20. Kitamura YI, Kitamura T, Kruse JP, Raum JC, Stein R, Gu W and Accili D: FoxO1 protects against pancreatic beta cell failure through NeuroD and MafA induction. Cell Metab 2: 153-163, 2005.

21. Kobayashi M,KikuchiO, Sasaki T, Kim HJ, Yokota-Hashimoto H, Lee YS, Amano K, Kitazumi T, Susanti VY, Kitamura YI, et al: FoxO1 as a double-edged sword in the pancreas: Analysis of pancreas- and $\beta$-cell-specific FoxO1 knockout mice. Am J Physiol Endocrinol Metab 302: E603-E613, 2012.

22. Gerst F, Kaiser G, Panse M, Sartorius T, Pujol A, Hennige AM, Machicao F, Lammers R, Bosch F, Häring HU, et al: Protein kinase $\mathrm{C} \delta$ regulates nuclear export of FOXO1 through phosphorylation of the chaperone 14-3-3 3 . Diabetologia 58: 2819-2831, 2015.

23. Kaiser G, Gerst F, Michael D, Berchtold S, Friedrich B, StrutzSeebohm N, Lang F, Häring HU and Ullrich S: Regulation of forkhead box O1 (FOXO1) by protein kinase B and glucocorticoids: Different mechanisms of induction of beta cell death in vitro. Diabetologia 56: 1587-1595, 2013.

24. Kitamura T, Nakae J, Kitamura Y, Kido Y, Biggs WH III, Wright CV, White MF, Arden KC and Accili D: The forkhead transcription factor Foxol links insulin signaling to Pdx1 regulation of pancreatic beta cell growth. J Clin Invest 110: 1839-1847, 2002.

25. Mo X, Wang X, Ge Q and Bian F: The effects of SIRT1/FoxO1 on LPS induced INS-1 cells dysfunction. Stress 22: 70-82, 2019.

26. Boughanem H, Cabrera-Mulero A, Millán-Gómez M, GarridoSánchez L, Cardona F, Tinahones FJ, Moreno-Santos I and Macías-González M: Transcriptional analysis of FOXO1, C/ EBP- $\alpha$ and PPAR- $\gamma 2$ genes and their association with obesityrelated insulin resistance. Genes 10: 706, 2019. doi.org/10.3390/ genes10090706.

27. Samuel VT, Choi CS, Phillips TG, Romanelli AJ, Geisler JG, Bhanot S, McKay R, Monia B, Shutter JR, Lindberg RA, et al: Targeting foxo1 in mice using antisense oligonucleotide improves hepatic and peripheral insulin action. Diabetes 55: 2042-2050, 2006.

28. Yang L, Li P, Fu S, Calay ES and Hotamisligil GS: Defective hepatic autophagy in obesity promotes ER stress and causes insulin resistance. Cell Metab 11: 467-478, 2010.

29. Rivera JF, Costes S, Gurlo T, Glabe CG and Butler PC: Autophagy defends pancreatic $\beta$ cells from human islet amyloid polypeptideinduced toxicity. J Clin Invest 124: 3489-3500, 2014.

30. Quan W, Hur KY, Lim Y, Oh SH, Lee JC, Kim KH, Kim GH, Kim SW, Kim HL, Lee MK, et al: Autophagy deficiency in beta cells leads to compromised unfolded protein response and progression from obesity to diabetes in mice. Diabetologia 55: 392-403, 2012.

31. Bachar-Wikstrom E, Wikstrom JD, Ariav Y, Tirosh B, Kaiser N, Cerasi E and Leibowitz G: Stimulation of autophagy improves endoplasmic reticulum stress-induced diabetes. Diabetes 62: 1227-1237, 2013.

32. Riahi Y, Wikstrom JD, Bachar-Wikstrom E, Polin N, Zucker H, Lee MS, Quan W, Haataja L, Liu M, Arvan P, et al: Erratum to: Autophagy is a major regulator of beta cell insulin homeostasis. Diabetologia 59: 1575-1576, 2016.

33. Yin JJ, Li YB, Wang Y, Liu GD, Wang J, Zhu XO and Pan SH: The role of autophagy in endoplasmic reticulum stress-induced pancreatic $\beta$ cell death. Autophagy 8: 158-164, 2012.

34. Priyadarshini M, Cole C, Oroskar G, Ludvik AE, Wicksteed B, $\mathrm{He} \mathrm{C}$ and Layden BT: Free fatty acid receptor 3 differentially contributes to $\beta$-cell compensation under high-fat diet and streptozotocin stress. Am J Physiol Regul Integr Comp Physiol 318: R691-R700, 2020.

35. Huang C, Wang HY, Wang ME, Hsu MC, Wu YS, Jiang YF, Wu LS, Jong DS and Chiu CH: Kisspeptin-activated autophagy independently suppresses non-glucose-stimulated insulin secretion from pancreatic $\beta$-cells. Sci Rep 9: 17451, 2019.

36. Zhou J, Kang X, Luo Y, Yuan Y, Wu Y, Wang M and Liu D: Glibenclamide-induced autophagy inhibits its insulin secretionimproving function in $\beta$ cells. Int J Endocrinol 2019: 1265175, 2019. doi.org/10.1155/2019/1265175. 
37. Yamamoto S, Kuramoto K, Wang N, Situ X, Priyadarshini M, Zhang W, Cordoba-Chacon J, Layden BT and He C: Autophagy differentially regulates insulin production and insulin sensitivity. Cell Rep 23: 3286-3299, 2018.

38. Ebato C, Uchida T, Arakawa M, Komatsu M, Ueno T, Komiya K, Azuma K, Hirose T, Tanaka K, Kominami E, et al: Autophagy is important in islet homeostasis and compensatory increase of beta cell mass in response to high-fat diet. Cell Metab 8: 325-332, 2008.

39. Jung HS, Chung KW, Won Kim J, Kim J, Komatsu M, Tanaka K, Nguyen YH, Kang TM, Yoon KH, Kim JW, et al: Loss of autophagy diminishes pancreatic beta cell mass and function with resultant hyperglycemia. Cell Metab 8: 318-324, 2008.

40. Galicia-Garcia U, Benito-Vicente A, Jebari S, Larrea-Sebal A, Siddiqi H, Uribe KB, Ostolaza H and Martín C: Pathophysiology of type 2 diabetes mellitus. Int J Mol Sci 21: 21, 2020

41. Frendo-Cumbo S, Jaldin-Fincati JR, Coyaud E, Laurent EMN, Townsend LK, Tan JMJ, Xavier RJ, Pillon NJ, Raught B, Wright DC, et al: Deficiency of the autophagy gene ATG16L1 induces insulin resistance through KLHL9/KLHL13/CUL3 mediated IRS1 degradation. J Biol Chem 294: 16172-16185, 2019

42. Chan YK, Sung HK, Jahng JW, Kim GH, Han M and Sweeney G: Lipocalin-2 inhibits autophagy and induces insulin resistance in H9c2 cells. Mol Cell Endocrinol 430: 68-76, 2016.

43. Guo Q, Xu L, Li H, Sun H, Liu J, Wu S and Zhou B: Progranulin causes adipose insulin resistance via increased autophagy resulting from activated oxidative stress and endoplasmic reticulum stress. Lipids Health Dis 16: 25, 2017. doi.org/10.1186/ s12944-017-0425-6.

44. Barlow AD and Thomas DC: Autophagy in diabetes: B-cell dysfunction, insulin resistance, and complications. DNA Cell Biol 34: 252-260, 2015.

45. Chen ZF, Li YB, Han JY, Wang J, Yin JJ, Li JB and Tian H: The double-edged effect of autophagy in pancreatic beta cells and diabetes. Autophagy 7: 12-16, 2011.

46. Buteau J and Accili D: Regulation of pancreatic beta-cell function by the forkhead protein FoxO1. Diabetes Obes Metab 9 (Suppl 2): 140-146, 2007.

47. Kitamura $\mathrm{T}$ and Ido Kitamura Y: Role of FoxO proteins in pancreatic beta cells. Endocr J 54: 507-515, 2007.

48. Talchai SC and Accili D: Legacy effect of Foxol in pancreatic endocrine progenitors on adult $\beta$-cell mass and function. Diabetes 64: 2868-2879, 2015.

49. Cai H, Jiang Z, Yang X, Lin J, Cai Q and Li X: Circular RNA HIPK 3 contributes to hyperglycemia and insulin homeostasis by sponging miR-192-5p and upregulating transcription factor forkhead box O1. Endocr J 67: 397-408, 2020.

50. Robertson RP, Harmon J, Tran PO, Tanaka Y and Takahashi H: Glucose toxicity in beta-cells: Type 2 diabetes, good radicals gone bad, and the glutathione connection. Diabetes 52: 581-587, 2003.

51. Zhang T, Kim DH, Xiao X, Lee S, Gong Z, Muzumdar R, CalabuigNavarro V, Yamauchi J, Harashima H, Wang R, et al: FoxO1 plays an important role in regulating $\beta$-cell compensation for insulin resistance in male mice. Endocrinology 157: 1055-1070, 2016.

52. Kibbe C, Chen J, Xu G, Jing G and Shalev A: FOXO1 competes with carbohydrate response element-binding protein (ChREBP) and inhibits thioredoxin-interacting protein (TXNIP) transcription in pancreatic beta cells. J Biol Chem 288: 23194-23202, 2013.

53. Kim HJ, Kobayashi M, Sasaki T, Kikuchi O, Amano K, Kitazumi T, Lee YS, Yokota-Hashimoto H, Susanti VY, Kitamura YI, et al: Overexpression of FoxO1 in the hypothalamus and pancreas causes obesity and glucose intolerance. Endocrinology 153: 659-671, 2012.

54. Zhang X, Yong W, Lv J, Zhu Y, Zhang J, Chen F, Zhang R, Yang T, Sun Y and Han X: Inhibition of forkhead box O1 protects pancreatic beta-cells against dexamethasone-induced dysfunction. Endocrinology 150: 4065-4073, 2009.

55. Kong $X$, Zhang L, Hua X and Ma X: Squamosamide derivative FLZ protects pancreatic $\beta$-cells from glucotoxicity by stimulating Akt-FOXO1 pathway. J Diabetes Res 2015: 803986, 2015.

56. Liu XD, Ruan JX, Xia JH, Yang SL, Fan JH and Li K: The study of regulatory effects of Pdx-1, MafA and NeuroD1 on the activity of porcine insulin promoter and the expression of human islet amyloid polypeptide. Mol Cell Biochem 394: 59-66, 2014.

57. Shao S, Liu Z, Yang Y, Zhang M and Yu X: SREBP-1c, Pdx-1, and GLP-1R involved in palmitate-EPA regulated glucose-stimulated insulin secretion in INS-1 cells. J Cell Biochem 111: 634-642, 2010
58. Zhang F, Ma D, Zhao W, Wang D, Liu T, Liu Y, Yang Y, Liu Y, Mu J, Li B, et al: Obesity-induced overexpression of miR-802 impairs insulin transcription and secretion. Nat Commun 11: 1822, 2020. doi: 10.1038/s41467-020-15529-w.

59. Kim DH, Zhang T, Ringquist $\mathrm{S}$ and Dong HH: Targeting FoxO1 for hypertriglyceridemia. Curr Drug Targets 12: 1245-1255, 2011.

60. Matsumoto M, Han S, Kitamura T and Accili D: Dual role of transcription factor FoxO1 in controlling hepatic insulin sensitivity and lipid metabolism. J Clin Invest 116: 2464-2472, 2006.

61. Wang S, Ai H, Liu L, Zhang X, Gao F, Zheng L, Yi J, Sun L, Yu C, Zhao $\mathrm{H}$, et al: Micro-RNA-27a/b negatively regulates hepatic gluconeogenesis by targeting FOXO1. Am J Physiol Endocrinol Metab 317: E911-E924, 2019.

62. Penniman CM, Suarez Beltran PA, Bhardwaj G, Junck TL, Jena J, Poro K, Hirshman MF, Goodyear LJ and O'Neill BT: Loss of FoxOs in muscle reveals sex-based differences in insulin sensitivity but mitigates diet-induced obesity. Mol Metab 30: 203-220, 2019.

63. Zhang W, Patil S, Chauhan B, Guo S, Powell DR, Le J, Klotsas A, Matika R, Xiao X, Franks R, et al: FoxO1 regulates multiple metabolic pathways in the liver: Effects on gluconeogenic, glycolytic, and lipogenic gene expression. J Biol Chem 281: 10105-10117, 2006

64. Zhang K, Li L, Qi Y, Zhu X, Gan B, DePinho RA, Averitt T and Guo S: Hepatic suppression of Foxol and Foxo3 causes hypoglycemia and hyperlipidemia in mice. Endocrinology 153: 631-646, 2012.

65. Du K, Herzig S, Kulkarni RN and Montminy M: TRB3: A tribbles homolog that inhibits Akt/PKB activation by insulin in liver. Science 300: 1574-1577, 2003.

66. Greer EL, Oskoui PR, Banko MR, Maniar JM, Gygi MP, Gygi SP and Brunet A: The energy sensor AMP-activated protein kinase directly regulates the mammalian $\mathrm{FOXO} 3$ transcription factor. J Biol Chem 282: 30107-30119, 2007.

67. Caselli C: Role of adiponectin system in insulin resistance. Mol Genet Metab 113: 155-160, 2014.

68. Yaghootkar H, Lamina C, Scott RA, Dastani Z, Hivert MF, Warren LL, Stancáková A, Buxbaum SG, Lyytikäinen LP, Henneman P, et al; GENESIS Consortium; RISC Consortium: Mendelian randomization studies do not support a causal role for reduced circulating adiponectin levels in insulin resistance and type 2 diabetes. Diabetes 62: 3589-3598, 2013.

69. Wang A, Li T, An P, Yan W, Zheng H, Wang B and Mu Y: Exendin-4 upregulates adiponectin level in adipocytes via Sirt1/ Foxo-1 signaling pathway. PLoS One 12: e0169469, 2017.

70. Sanchez AM, Bernardi H, Py G and Candau RB: Autophagy is essential to support skeletal muscle plasticity in response to endurance exercise. Am J Physiol Regul Integr Comp Physiol 307: R956-R969, 2014.

71. Jash S and Puri V: FoxO1-autophagy axis regulates lipid droplet growth via FSP27. Cell Cycle 15: 2856-2857, 2016.

72. Zhao Y, Yang J, Liao W, Liu X, Zhang H, Wang S, Wang D, Feng J, Yu L and Zhu WG: Cytosolic FoxO1 is essential for the induction of autophagy and tumour suppressor activity. Nat Cell Biol 12: 665-675, 2010.

73. Liu L, Zheng LD, Zou P, Brooke J, Smith C, Long YC, Almeida FA, Liu D and Cheng Z: FoxO1 antagonist suppresses autophagy and lipid droplet growth in adipocytes. Cell Cycle 15: 2033-2041, 2016

74. Ren H, Shao Y, Wu C, Ma X, Lv C and Wang Q: Metformin alleviates oxidative stress and enhances autophagy in diabetic kidney disease via AMPK/SIRT1-FoxO1 pathway. Mol Cell Endocrinol 500: 110628, 2020.

75. Wang J, Shen L, Hong H,Li J, Wang H and Li X: Atrasentan alleviates high glucose-induced podocyte injury by the microRNA-21/ forkhead box O1 axis. Eur J Pharmacol 852: 142-150, 2019.

76. Guo X, Lin H, Liu J, Wang D, Li D, Jiang C, Tang Y, Wang J, Zhang T, Li Y, et al: 1,25-Dihydroxyvitamin D attenuates diabetic cardiac autophagy and damage by vitamin $\mathrm{D}$ receptormediated suppression of FoxO1 translocation. J Nutr Biochem 80: 108380, 2020.

77. Sengupta A, Molkentin JD and Yutzey KE: FoxO transcription factors promote autophagy in cardiomyocytes. J Biol Chem 284: 28319-28331, 2009. 
78. Ning Y, Li Z and Qiu Z: FOXO1 silence aggravates oxidative stress-promoted apoptosis in cardiomyocytes by reducing autophagy. J Toxicol Sci 40: 637-645, 2015.

79. Wang B, Yang Q, Sun YY, Xing YF, Wang YB, Lu XT, Bai WW, Liu XQ and Zhao YX: Resveratrol-enhanced autophagic flux ameliorates myocardial oxidative stress injury in diabetic mice. J Cell Mol Med 18: 1599-1611, 2014.

80. Li Y, Jiang J, Liu W, Wang H, Zhao L, Liu S, Li P, Zhang S, Sun $\mathrm{C}, \mathrm{Wu}$ Y, et al: microRNA-378 promotes autophagy and inhibits apoptosis in skeletal muscle. Proc Natl Acad Sci USA 115: E10849-E10858, 2018.

81. Yang M, Lin Y, Wang Y and Wang Y: High-glucose induces cardiac myocytes apoptosis through Foxo1/GRK2 signaling pathway. Biochem Biophys Res Commun 513: 154-158, 2019.

82. Liu HY, Han J, Cao SY, Hong T, Zhuo D, Shi J, Liu Z and Cao W: Hepatic autophagy is suppressed in the presence of insulin resistance and hyperinsulinemia: Inhibition of FoxO1-dependent expression of key autophagy genes by insulin. J Biol Chem 284: 31484-31492, 2009.
83. Xiong X, Tao R, DePinho RA and Dong XC: The autophagyrelated gene 14 (Atg14) is regulated by forkhead box $\mathrm{O}$ transcription factors and circadian rhythms and plays a critical role in hepatic autophagy and lipid metabolism. J Biol Chem 287: 39107-39114, 2012.

84. Lettieri Barbato D, Tatulli G, Aquilano K and Ciriolo MR: FoxO1 controls lysosomal acid lipase in adipocytes: implication of lipophagy during nutrient restriction and metformin treatment. Cell Death Dis 4: e861, 2013. doi.org/10.1038/cddis.2013.404. International (CC BY-NC-ND 4.0) License. 